\title{
Réduire la transmission du SRAS-CoV-2 par voie aérienne
}

\author{
Sarah Addleman MDCM, Victor Leung MD, Leyla Asadi MD MSP, Abdu Sharkawy MD, Jennifer McDonald MD
}

Citation : CMAJ 2021 July 5;193:E1010-1. doi : 10.1503/cmaj.210830-f; diffusion hâtive le 8 juin 2021

Voir la version anglaise de l'article ici : www.cmaj.ca/lookup/doi/10.1503/cmaj.210830

\section{A} u début de la pandémie de COVID-19, notre compréhension de la transmission des virus respiratoires était basée sur un cadre conceptuel qui distinguait 2 types de particules infectieuses: les gouttelettes de $>5 \mu \mathrm{m}$, qui se déposent près de leur source, et les particules aérosolisées de $<5 \mu \mathrm{m}$, qui restent en suspension dans l'air. Or, les chercheurs du domaine des aérosols contestaient cette catégorisation traditionnelle, sachant que des particules pouvant atteindre $100 \mu \mathrm{m}$ de diamètre peuvent demeurer en suspension dans l'air et être inhalées ${ }^{1}$. L'Agence de la santé publique du Canada (ASPC) a reconnu en novembre 2020 que la transmission par aérosols du SRAS-CoV-2 était possible, et de récentes mises à jour de l'Organisation mondiale de la Santé $(\mathrm{OMS})^{2}$ et des Centers for Disease Control and Prevention (CDC ${ }^{3}$ des États-Unis font mention de la transmission par aérosols sur de courtes et de longues distances. Malgré cela, les directives de santé publique canadiennes n'ont pas été mises à jour pour tenir compte de la transmission par voie aérienne, probablement parce que son importance relative par rapport à d'autres modes de transmission ne fait pas encore consensus. Nous croyons qu'il n'est pas nécessaire d'atteindre un consensus sur la prépondérance de la transmission par voie aérienne avant d'apporter des changements significatifs aux directives de santé publique canadiennes. Nous demandons que les directives et les politiques sur la ventilation et le port du couvre-visage soient mises à jour et qu'elles soient le fruit d'une collaboration multidisciplinaire afin de garantir les normes de sécurité les plus élevées pour les collectivités canadiennes.

Selon les CDC, le SRAS-CoV-2 se propage par l'inhalation d'aérosols infectieux en suspension dans l'air, par contact avec des gouttelettes s'étant déposées sur des surfaces (éclaboussures et gouttelettes pulvérisées) et par contact avec des surfaces; l'inhalation d'aérosols, même si elle est plus probable à courte distance parce que les gouttelettes y sont plus concentrées, peut aussi se produire à plus longue distance selon les conditions environnementales ${ }^{3}$. La possibilité de transmission du SRAS-CoV-2 par voie aérienne a été montrée par la présence de cas de transmission asymptomatique, de superpropagation,

\section{POINTS CLÉS}

- La transmission du SRAS-CoV-2 par inhalation d'aérosols à courte et à longue distance, aussi appelée transmission par voie aérienne, est reconnue par des agences de santé publique internationales.

- Les directives et les politiques canadiennes en matière de santé publique devraient être mises à jour pour tenir compte de ce mode de transmission.

- La ventilation est une mesure clé de réduction de la transmission par voie aérienne; les entreprises et les écoles devraient bénéficier de recommandations et d'un financement leur permettant de procéder aux évaluations et aux améliorations qui s'imposent.

- Les directives concernant le port d'équipement de protection individuelle par les travailleurs de la santé et les autres travailleurs essentiels gagneraient à être revues de façon à promouvoir le port des respirateurs N95 à plus grande échelle.

- Pour lutter contre la transmission par voie aérienne, il faut avoir recours à l'expertise d'équipes interdisciplinaires qui pourront trouver des solutions afin de stopper plus rapidement la pandémie.

de transmission à distance confirmée par la recherche des contacts et le séquençage et d'infection nosocomiale malgré le respect scrupuleux des directives de port d'équipement de protection individuelle (EPI) contre les contacts et les gouttelettes, ainsi que par des études chez l'animal attestant d'une transmission par des conduits de ventilation ${ }^{4}$. Bien que le prélèvement d'échantillons d'air visant la détection de pathogènes vivants hors des laboratoires soit difficile (les chercheurs ont échoué dans le cas de la rougeole et de la tuberculose, 2 maladies transmises par voie aérienne), la présence de SRAS-CoV-2 viable a été détectée dans l'air d'une chambre d'hôpital et celui d'une voiture conduite pendant 15 minutes par un patient légèrement symptomatique ${ }^{4}$. Notons que la transmission de la tuberculose se fait principalement par contact étroit, mais cette maladie peut aussi se transmettre à distance. 
Comme il a été établi qu'une infection au SRAS-CoV-2 est possible par inhalation d'aérosols à courte et à longue distance, l'utilisation de l'expression "par voie aérienne » désigne adéquatement le potentiel de transmission du virus par l'air et permet de clarifier les messages à la population. L'importance de la ventilation, de la filtration de l'air et du port du couvre-visage à l'intérieur, même à distance, et de la tenue de rassemblements à l'extérieur prend tout son sens si on reconnaît explicitement l'existence de la transmission par voie aérienne.

La ventilation joue un rôle clé dans la réduction de la transmission par aérosols sur une longue distance; il faudrait donc diffuser à grande échelle des recommandations pratiques et offrir du financement pour prioriser l'évaluation et l'amélioration de la ventilation dans les entreprises et les écoles. Les mesures d'atténuation pour lesquelles les preuves sont insuffisantes, comme la désinfection massive et l'installation de plexiglas protégeant contre la propagation de gouttelettes, continuent d'être des investissements peu judicieux qui procurent un faux sentiment de sécurité. Le risque de transmission par fomites est jugé faible, et le plexiglas peut nuire à la circulation de l'air et favoriser la transmission par aérosols 5 . Les $\mathrm{CDC}$ ont publié une trousse à outils qui propose diverses interventions pour améliorer la ventilation dans les bâtiments, dont plusieurs interventions facilement applicables et relativement abordables $^{6}$. Santé Canada a récemment mis à jour ses directives d'évaluation et d'amélioration de la qualité de l'air dans les logements au moyen du taux de dioxyde de carbone ${ }^{7}$, un indicateur indirect de la qualité de la ventilation facilement mesurable. II faut de toute urgence formuler des directives similaires pour les espaces intérieurs, en particulier les écoles ${ }^{8}$; un nombre croissant de données montrent qu'en plus de conférer une protection contre la transmission par voie aérienne, une bonne ventilation a des effets positifs sur la santé en général et la santé cognitive.

Il a été démontré que le personnel soignant ayant des contacts étroits et prolongés avec les patients est particulièrement à risque de contracter une infection par le SRAS-CoV-2, malgré le port d'EPI contre la transmission par contact et gouttelettes ${ }^{9}$. Les grandes éclosions survenues dans des établissements de soins de longue durée ont été le résultat d'une combinaison parfaite de conditions défavorables : nombreux résidents en phase présymptomatique ou symptomatique précoce - durant lesquelles la contagion est importante -, et milieux où la ventilation est moins bonne que dans les hôpitaux modernes. Ces éclosions montrent que le fait de se contenter d'appliquer des mesures qui ne ciblent que les contacts et les gouttelettes peut avoir un effet dévastateur dans les vieux établissements de soins. L'intensité des taux d'infection dans les établissements de soins de longue durée s'explique mal par de simples erreurs de manipulation de l'EPI; il serait donc sage d'insister davantage sur la qualité des masques et sur la ventilation (y compris dans les salles de repos des employés).

Bien qu'une bonne ventilation puisse atténuer le risque de transmission du SRAS-CoV-2 par aérosols sur une longue distance, la reconnaissance de la propagation par voie aérienne sur de courtes distances par l'OMS et les CDC montre qu'il est urgent de revoir rapidement les directives concernant l'EPI dans tous les milieux de soins au Canada. Les masques de grande qualité, comme les respirateurs N95 ne sont actuellement obligatoires que pour les « interven- tions médicales pouvant générer des aérosols ». Cette directive reconnaît que les respirateurs N95 sont le meilleur moyen de protection contre les aérosols à courte distance. Les masques chirurgicaux offrent un contrôle à la source et une certaine protection contre les aérosols ${ }^{10}$, mais leur efficacité contre l'inhalation d'aérosols est très variable. Selon des études d'observation, le taux d'infection par le SRAS-CoV-2 est plus élevé chez les travailleurs de la santé qui utilisent des masques chirurgicaux que chez ceux qui utilisent des respirateurs ${\mathrm{N} 95^{9}}^{\circ}$. De plus, les CDC recommandent le port de respirateurs N95, de blouses, de gants et d'équipement de protection oculaire lors de la prestation de soins à des patients ayant une infection au SRAS-CoV-2 confirmée ou présumée ${ }^{11}$. Par ailleurs, des études récentes ont montré que les activités vigoureuses et la toux produisent plus d'aérosols que certaines interventions médicales pouvant en générer ${ }^{12}$. Les travailleurs de la santé canadiens, quel que soit leur environnement de travail, devraient toujours avoir accès aux respirateurs N95 et être informés que ces derniers ne sont pas réservés uniquement aux interventions médicales pouvant générer des aérosols; cette suggestion s'harmonise avec un récent message de l'ASPC ${ }^{13}$.

À l'extérieur des établissements de santé, les travailleurs essentiels œuvrant dans des milieux de forte proximité, comme les centres de transformation de la viande, les usines et les centres de distribution, ont été touchés de manière disproportionnée par la COVID-19. Bien que les mesures sociales comme les congés de maladie payés soient vitales, le port de masques standardisés (idéalement des respirateurs ajustés) demeure une mesure de protection indispensable. On craint moins à présent une pénurie de respirateurs N95, dont la production locale a augmenté, y compris celle de modèles confortables permettant un ajustement plus universel. De plus, les dispositions qui permettent le retrait des masques à l'intérieur si une distanciation de 2 mètres peut être respectée ne sont pas bien pensées, car elles ne tiennent pas compte de la transmission par voie aérienne dans un espace clos et continuent d'exposer les travailleurs essentiels et la population à un risque.

Nous avons une expertise canadienne en génie et en hygiène du travail qui nous permet d'orienter les politiques relatives à la ventilation et au port du couvre-visage. Même si l'accès à des vaccins hautement efficaces permet d'entrevoir la fin de la pandémie de COVID-19 au Canada, une collaboration multidisciplinaire pourrait accélérer le processus. Il a fallu des décennies pour que la communauté médicale accepte que la tuberculose et la rougeole se transmettent par voie aérienne. Or, les connaissances sur la transmission du SRAS-CoV-2 par voie aérienne ont rapidement progressé; il est temps que les directives et les politiques de santé publique canadiennes en tiennent compte.

\section{Références}

1. Tang JW, Bahnfleth WP, Bluyssen PM, et al. Dismantling myths on the airborne transmission of severe acute respiratory syndrome coronavirus (SARS-CoV-2). J Hosp Infect. 2021;110:89-96.

2. Coronavirus disease (COVID-19): How is it transmitted? [communiqué de presse]. Geneva: World Health Organization; modified 2021 Apr. 30. Accessible ici : https://www.who.int/news-room/q-a-detail/coronavirus-disease-covid -19-how-is-it-transmitted (consulté le 2 mai 2021). 
3. Scientific brief: SARS-CoV-2 Transmission. Atlanta: Centers for Disease Control; updated 2021 May 7. Accessible ici : https://www.cdc.gov/ coronavirus/2019-ncov/science/science-briefs/sars-cov-2-transmission.html (consulté le 22 mai 2021).

4. Greenhalgh T, Jimenez JL, Prather KA, et al. Ten scientific reasons in support of airborne transmission of SARS-CoV-2. Lancet 2021;397:1603-5.

5. Lessler J, Grabowski MK, Grantz KH, et al. Household COVID-19 risk and inperson schooling. Science 2021 Apr. 29 [cyberpublication avant impression]. doi: $10.1126 /$ science.abh2939.

6. Ventilation in buildings. Atlanta: Centers for Disease Control; modified 2021 Mar. 23. Accessible ici : https://www.cdc.gov/coronavirus/2019-ncov/ community/ventilation.html\#considerations (consulté le 22 mai 2021).

7. Recommended long-term exposure limit. In: Residential Indoor Air Quality Guidelines: Carbon Dioxide. Ottawa: Health Canada; modified 2021 Apr. 23. Accessible ici : https://www.canada.ca/en/health-canada/services/ publications/healthy-living/residential-indoor-air-quality-guidelines -carbon-dioxide.html\#sec71 (consulté le 22 mai 2021).

8. Gettings J, Czarnik M, Morris E, et al. Mask use and ventilation improvements to reduce COVID-19 incidence in elementary schools - Georgia, November 16-December 11, 2020. MMWR Morb Mortal Wkly Rep 2021;70:779-84.
9. Chou R, Dana T, Buckley DI, et al. Epidemiology of and risk factors for coronavirus infection in health care workers: a living rapid review. Ann Intern Med 2020;173:120-36.

10. Cheng Y, Ma N, Witt C, et al. Face masks effectively limit the probability of SARS-CoV-2 transmission. Science 2021 May 20 [cyberpublication avant impression]. doi: 10.1126/science.abg6296.

11. Interim infection prevention and control recommendations for healthcare personnel during the coronavirus disease 2019 (COVID-19) pandemic. Atlanta: Centers for Disease Control; modified 2021 Feb. 23. Accessible ici: https://www.cdc.gov/coronavirus/2019-ncov/hcp/ infection-control-recommendations.html (consulté le 22 mai 2021).

12. Hamilton F, Arnold D, Bzdek BR, et al. Aerosol generating procedures: Are they of relevance for transmission of SARS-CoV-2? Lancet Respir Med 2021 May 6 [cyberpublication avant impression]. doi: 10.1016/S22132600(21)00216-2.

13. Njoo H. Response from the Public Health Agency of Canada [lettre]. Ottawa: Public Health Agency of Canada; 2021 Mar. 24. Accessible ici : https://www.wigmorising.ca/wp-content/uploads/2021/04/ Response-from-the-Public-Health-Agency-of-Canada.pdf (consulté le 2 mai 2021).
Intérêts concurrents : Leyla Asadi a déclaré avoir reçu une subvention de l'Alberta Health Innovates Clinician Fellowship. Aucun autre intérêt concurrent déclaré.

Cet article a été révisé par des pairs.

Affiliations : Département de médecine d'urgence (Addleman), Université d'Ottawa, Ottawa, Ont.; Division de microbiologie médicale (Leung), Département de pathologie et de médecine de laboratoire et Division des maladies infectieuses (Leung), Département de médecine, Université de la ColombieBritannique, Vancouver, C-B.; Département de médecine (Asadi), Université de l'Alberta,
Edmonton, Alb.; Division des maladies infectieuses (Sharkawy), Département de médecine, Université de Toronto, Toronto, Ont.; Division de physiatrie et de réadaptation (McDonald), Département de médecine, Université d'Ottawa, Ottawa, Ont.

Collaborateurs : Sarah Addleman et Jennifer McDonald ont contribué à l'élaboration et à la conception des travaux et ont rédigé l'ébauche du manuscrit. Tous les auteurs ont révisé de façon critique le contenu intellectuel important du manuscrit; ils ont donné leur approbation finale pour la version destinée à être publiée et assument l'entière responsabilité de tous les aspects du travail.
Propriété intellectuelle du contenu : Il s'agit d'un article en libre accès distribué conformément aux modalités de la licence Creative Commons Attribution (CC BY-NC-ND 4.0), qui permet l'utilisation, la diffusion et la reproduction dans tout médium à la condition que la publication originale soit adéquatement citée, que l'utilisation se fasse à des fins non commerciales (c.-à-d., recherche ou éducation) et qu'aucune modification ni adaptation n'y soit apportée. Voir : https://creativecommons.org/ licenses/by-nc-nd/4.0/deed.fr.

Correspondance : Sarah Addleman, saddlemanfrankel@toh.ca 